\title{
On the Issue of the Influence of Interfacial Interactions on the Filtration Processes in Coal
}

\author{
Ivan Elkin ${ }^{1, *}$, and Yunliang Tan ${ }^{2}$ \\ ${ }^{1}$ T. F. Gorbachev Kuzbass State Technical University, Department of Physics, 650099, Kemerovo \\ Russia \\ ${ }^{2}$ State Key Laboratory of Mining Disaster Prevention and Control, Shandong University of Science \\ and Technology, Qingdao 266590, China
}

\begin{abstract}
The features of gas-liquid fluid filtration processes in a coal mass are discussed and general patterns of mass transfer, extending previously known concepts, are established in the article. It indicates the degree of the influence of wettability, contact angle of wetting on filtration processes. It is assumed the coal-liquid-gas interfacial interactions when infusing the coal mass lead to the deformation of capillary pore space of coal, splitting, swelling and clogging of filter pores and capillaries. A comparison is made between the thermal effect and the effect of surfactants on the deformation processes of the capillary-porous structure of coal. Filtration processes are described by the gas-liquid fluid mass transfer equations. Coal-liquid-gas interfacial processes lead to a change in the state of the interacting phases, which leads to a change in both the filtration properties of the liquid and the filtration properties of a capillary pore space, which is especially characteristic of coal in relation to other rocks. Investigating the interfacial interactions when filtering in the laboratory, it is possible to determine the functions of the variation of the key coefficients in the mass transfer equations. On the basis of such studies, it is possible to predict the results of mass transfer in a coal mass being infused or degassed, as well as the change in the coal mass stress state.
\end{abstract}

\section{Introduction}

Modern technologies are aimed at achieving maximum safety of mining, where one of the main components is to control the stress state, to prevent dust forming and gas emission. These activities are closely related to the mass transfer control in the coal mass [1-7]. The phenomenon of mass transfer in a coal mass is closely connected with the technology of pre-moistening of a coal seam, degassing of coal mass, and gas production. The water, fluid infusion into a coal seam has many purposes: controlling the dynamic state of a coal seam, dust suppression, changes in the stress state, and gas-dynamic state of a coal seam $[8,9]$.

In practice, the effective moistening radius is evaluated the formula [4]:

$$
R_{\mathrm{w}}=31.6 \sqrt{Q_{T} t / \pi h N \gamma}
$$

\footnotetext{
* Corresponding author: jelkin@mail.ru
} 
where $N=10\left(W_{\max }-W_{e}\right)$ - moistening rate, $1 / \mathrm{t} ; W_{\max }$ - maximum hygroscopic moisture, $\% ; W_{e}$ - natural moisture, $\% ; Q_{T}$ - infusion rate, $\mathrm{m}^{3} / \mathrm{h} ; t$ - infusion time, $\mathrm{h} ; h$ - seam thickness, $\mathrm{m} ; \gamma$ - bulk density of coal, $\mathrm{t} / \mathrm{m}^{3} ; \pi=3.14$.

Practical studies show that the evaluations by formula (1) estimate only approximately the moistening radius. The given formula does not take into account the peculiarities of the interaction of coal with water, wetting processes, coal-liquid-gas interfacial interactions, and a change in the structure of a capillary-pore body during wetting.

In connection with these circumstances, it is relevant to study the processes of clogging filter pores during coal moistening, the establishment of patterns of deformation of the capillary pore space of coal during interfacial interactions. The clogging occurs due to the interaction of water molecules with coal molecules, the establishment of intermolecular interactions between liquid and coal molecules, which is also relevant for research.

\section{Research materials and methods}

For experimental studies, coals of various grades, various degrees of metamorphism, physicochemical properties, different moisture and structural features, porosity, strength characteristics, as well as distilled water and solutions of various surfactants were used. The surfactants used in mining industry were "Neolas", "Elfor", etc. Coal with a moisture content corresponding to dry, air-dry, natural moisture, maximum moisture was mostly used in research

The basic law describing filtration in capillary-porous body is the Darcy law $[10,11]$ :

$$
Q=K_{p} \frac{S}{\eta} \frac{\Delta P}{\Delta l}
$$

where $Q$ - filtering fluid flow rate, $\mathrm{m}^{3} / \mathrm{s} ; \Delta H$ - change in the pressure of the liquid when it passes the distance $\Delta l$ in the rock, $\mathrm{m} ; \Delta P$ - change in fluid pressure when it passes the distance $\Delta l$ in the rock, $\mathrm{Pa} ; K_{p}$ - porous medium phase permeability, $\mathrm{m}^{2} ; S$ - cross-section of the rock through which the fluid is filtered, $\mathrm{m}^{2}$.

In equation (2), the key variable characterizing the filtration properties of a capillaryporous body is the permeability. The study of changes in this variable when the filtering conditions change is one of the goals of this work.

A feature of the developed test rig for research in filtration processes is the low pressure of the filtering fluid. The fluid pressure is set so that it is possible to observe the phenomenon of filtration and the influence of interfacial interactions on the filtration processes, to establish the patterns of the observed phenomena. The main pressure of the liquid through the coal sample is established by hydrostatic pressure, determined by the difference in the level of the reservoir with the liquid under investigation relative to the level of the sample.

The rig diagram is shown in Fig. 1. The mass of samples for microfiltration studies was $100-200 \mathrm{~g}$. The developed method for studying the filtration properties of coal is based on a model of the coal seam wetting process in mine conditions. 


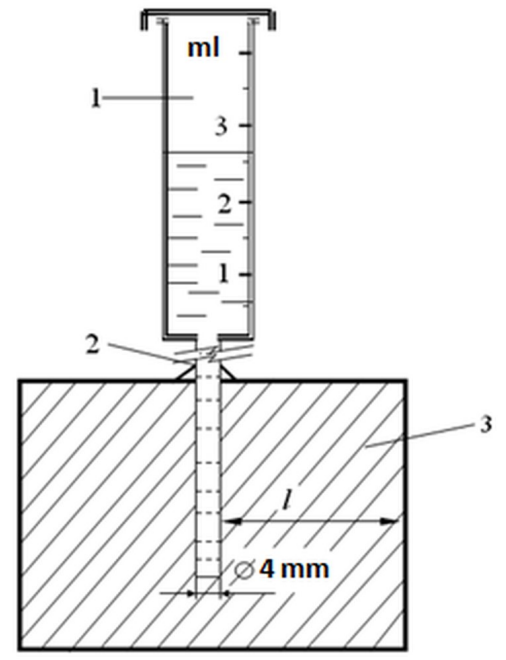

Fig.1. Scheme of the test rig: 1 - the test liquid tank; 2 - PVC connecting tube; 3 - coal sample.

The coal mass is a body with an inhomogeneous capillary-porous structure saturated with a gas-hydrate mixture, which is in dynamically changing conditions during mining operations. Changes in external conditions will determine the nature and direction of the flow of complex gas-dynamic processes in the mass, the mass transfer of liquid and gas, fluid.

In describing the movement of liquid and gas in dispersed media, differential equations of mass transfer are used. For a two-phase system, they will have the form [8]:

1) for gas

$$
m \frac{\partial}{\partial t}\left(v \rho_{g}\right)=-\operatorname{div}\left(\rho_{g} \vec{v}_{g}\right)+I_{g}
$$

2) for the liquid phase

$$
m \frac{\partial}{\partial t}\left(s \rho_{w}\right)=-\operatorname{div}\left(\rho_{w} \overrightarrow{\mathrm{v}}_{w}\right)+I_{w},
$$

where $v, s$ - bulk gas and water saturation; $I_{g}, I_{w}$ - source of liquid or gas or drain; $\rho_{g}$, $\rho_{w}$ - density of gas and liquid; $m$ - porosity; $v_{g}, v_{w}$ - liquid and gas velocity.

The mass transfer equations have a simple analytical solution for isotropic dispersion media. The solution to the problem of filtering a liquid in a gas-saturated solid becomes more complicated if a solid has a poly capillary structure [8]. At the same time, the pore size distribution for natural conditions is an uneven distribution depending on the initial mining and geological conditions of formation and condition of the coal mass, as well as dynamic processes in the mass as a result of technological work. The distribution of pores over the radii in the mass creates additional non-uniformity during filtration [8].

At high velocity of the fluid, in the first approximation, the kinetic processes at the gasto-coal interface are not taken into account, i.e. there are no processes of sorption and desorption, there is no hysteresis of gas adsorption, liquid on the surface of coal [9].

To solve the boundary value problem, the boundary and initial conditions for pressure are added to the system of equations (3) - (4). With a non-stationary process of moistering, many of the coefficients in equations (3) - (4) change depending on external conditions, 
effects, or other factors. These dependences can be represented as a set of empirical functions resulting from field observations or laboratory studies.

\section{Results}

Conducted laboratory studies with various coals showed the complexity of the filtration of liquids in coal. The main reason for this is the simultaneous occurrence of many phenomena.

The results are presented in Fig. 2 in the form of dependences of the flow rate $Q$ on time. Research results show that the flow of water through the sample varies significantly over time. The experimental data on the flow from time to time are well approximated by the formula in the form:

$$
Q(t)=Q_{0}\left(1+A e^{-\beta \cdot t}\right),
$$

where $A, \beta$ - empirical constants; $Q_{0}$ - limit value of consumption.

A significant factor influencing the type of functional dependence (5) is the initial moisture of coal. An increase in the initial moisture leads to a decrease in the empirical coefficient $A$ from 2 to 1 with a change in the moisture content of coal from 0 to $2 \%$. This reduces the period of unsteady filtering.

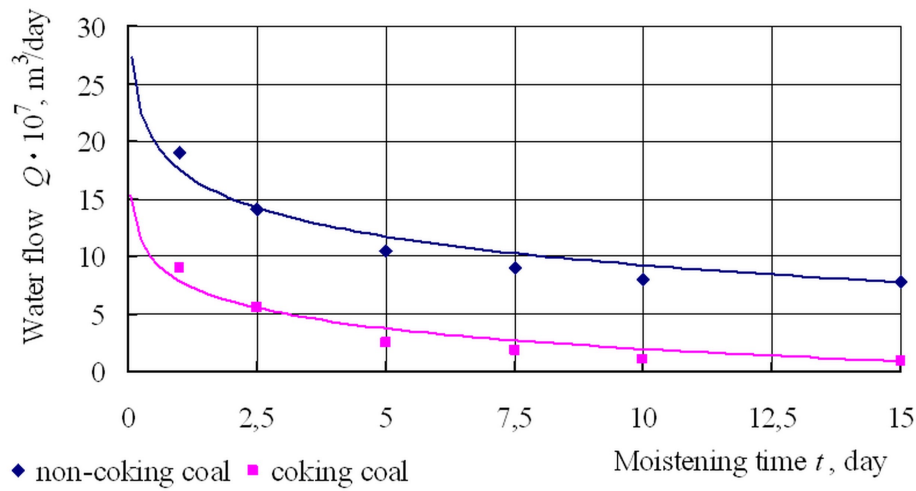

Fig. 2. The change in fluid flow during filtration through coal.

On the one hand, this is connected with the transition from the unsteady character of filtration to the established one due to an increase in the radius of liquid distribution in accordance with Darcy law, and, on the other hand, with the process of pore clogging due to interfacial interactions.

\section{Discussion}

From the calculations carried out by formulas (1) - (2), it was shown that the filtration wetting angle and wettability influence the filtration processes.

Diffusion of liquid molecules through the interface from the liquid phase into the intermolecular space of coal leads to a change in the state of all components of the intermolecular interaction and subsequently affects the filtration, mainly, on the effective cross section of the capillary radius. By the effective radius of the capillary is meant the radius of the section of the capillary, in which the filtration is described by the Poiseuille law. 
Thus, the intermolecular interaction at the interface in capillary pore space results in the effective capillary radius changes. The changes in the capillary radius depend on the time of interfacial interaction, the speed of the mass transfer process at the interface. At the same time, the properties of the surface change due to the adsorption of liquid molecules on the surface of a solid.

Unsteady filtration is observed at the initial moment of fluid infusion into the well. At this moment there is a significant change in the ratio between the liquid and the gas, the replacement of the gaseous phase with the liquid. The relationship between the changes in wettability, as a fluid with new properties, leads to a change in the filtration conditions.

The action of Laplace forces, the forces of capillary interaction is determined by the radii of capillaries. With a decrease in the capillary radius, the influence of the Laplace forces increases, an additional capillary pressure arises in the fluid according to the law.

$$
p_{k}=\frac{2 \sigma}{r} \cos \Theta,
$$

where $\sigma$ - is the coefficient of the surface tension of the liquid; $\Theta$ - contact angle of wetting; $r$ - the capillary radius.

As can be seen, a decrease in the capillary radius leads to a significant change in its filtration capacity.

The total pressure in the capillary is determined

$$
p=p_{n}+p_{k}
$$

where $p_{n}$ - is the pressure created by the infusion pump.

Given the large number of pores of different radii, the capillary effect on the filtration process becomes significant for capillaries with a size of less than $10^{-6} \mathrm{~m}$. For capillaries less than $10^{-8} \mathrm{~m}$, Knudsen filtration is observed. A feature in this case is the movement of the molecular layer under the action of diffuse forces, where the intermolecular interaction takes a more significant significance. The speed of such filtration is about $10^{-6} \mathrm{~m} / \mathrm{s}$, which corresponds to the diffusion movement of liquid molecules.

For macropores, where the radius of the capillaries is $10^{-4}-10^{-2} \mathrm{~m}$, the movement of gas-liquid fluid is determined by the pressure of the fluid and is more consistent with the Puisel equation.

The conducted model calculations have shown that wettability has a significant effect on mass transfer processes in coal and in coal seams. A separate capillary being considered; some important conclusions can be made. The velocity plots in the capillary take on the form shown in fig. 3 .

When the wettability is changed due to the adsorption of the hydrophilic molecular layer, the capillary velocity curve varies accordingly. In this case, the maximum filtration rate is achieved at a certain minimum thickness of the hydrophilic film on the surface of the capillary.

As noted in the general concepts $[1,2,4]$, the capillary radius is considered constant for any interfacial interactions in the angle. In technological and engineering calculations it is assumed that the volume of the capillary-porous system is constant, and its change does not affect the processes of mass transfer. Estimation of the radius of moistening from a single well is made by the given formula (1).

The range of coal moisture content being from 0 to $1 \%$; a significant decrease in consumption is observed, which is caused by low wettability of the surface, the absence of an adsorbed hydrophilic layer on the surface of the coal. 
On the other hand, a reduction in the flow rate in a capillary-porous body depends on the interfacial interactions, the speed, and the intensity of the interfacial process. The formation of an adsorbed liquid layer on the capillary surface is a major factor in which the effective capillary radius decreases to values at which filtration through this capillary will be difficult. Film formation is associated with the adsorption of liquid molecules on the surface of a capillary. In addition, the resulting film has the property of wedging the surface of a solid, which leads to the expansion of coal due to the effect of the Rehbinder effect.

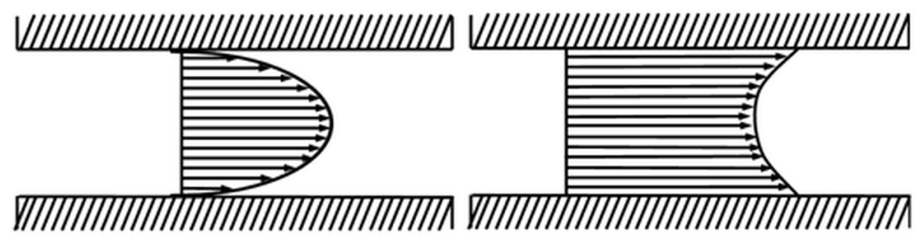

a

b

Fig. 3. Curves of velocity of fluid in the capillary: a - under pressure filtration in a hydrophobic capillary; $b$ - under filtration in a hydrophilic capillary.

The whole process of the coal mass moistening can be viewed not as stationary filtration in classical representations, but as unsteady filtering. In which the variables of the filtration process and the properties of a solid change significantly, the structure changes, the geometry of a capillary-porous space, where the structure of a capillary-porous body, from our point of view, is described by the functional dependence of the capillary radius distribution [6].

The effective radius of the capillary is somewhat different from the actual radius of the capillary formed by the surface of the capillary solid. Film thickness depends on adsorption processes, adhesion, intermolecular interactions at the interface.

Our additional studies on the expansion of coal under the influence of wetting with surfactant solutions show a non-uniform, non-linear increase in the size of the coal.

In our experiments, a comparison was made of the effect of heat exposure and the effect of the filtering liquid on the change in the structure and geometric dimensions of coal samples. Some of the results are shown in Fig. 4. The expansion versus time for coal is shown in Fig. 4. If the thermal effect on coal samples is characterized by a linear dependence of the expansion of coal over time, then non-linear dependence is observed for expansion during wetting and filtration with the participation of interfacial interaction. For thermal effects, linear expansion is described by the classical law in the form $l=l_{0}(1+\alpha T)$, where $l_{0}$ - is the initial linear size of the sample; $\alpha-$ is the linear thermal expansion coefficient; $T$ - temperature.

The determined basic linear thermal expansion coefficient for non-coking coals was $39.8 \cdot 10^{-6} \mathrm{~K}^{-1}$ in the temperature range from 20 to $100^{\circ} \mathrm{C}$ at the moisture content of the coal corresponding to the dry state.

During wetting and interfacial interaction of coal-liquid, non-linear time dependence is described by the formula in the form:

$$
l=l_{0}\left(1-e^{-\delta t}\right)
$$

where $l_{0}$ - is the initial linear size of the sample; $\delta$ - is a constant characterizing interfacial interactions; $t$ - is time.

According to the measurement results, it is shown that when the temperature changes by $30^{\circ} \mathrm{C}$, the expansion of coal is equivalent to the same expansion when the humidity increases from 0.1 to $2 \%$ under the action of the forces of capillary self-propulsion of the 
liquid. Similar studies with other porous materials showed a significantly lower value of the coefficient of expansion and the equivalent expansion of the interfacial interaction. In this case, the magnitude of the strength of the material and the specific surface area of the capillary-porous system of a solid matter are important.

The results show how interfacial interactions affect the change in the geometric structure of a capillary-porous system of coal.

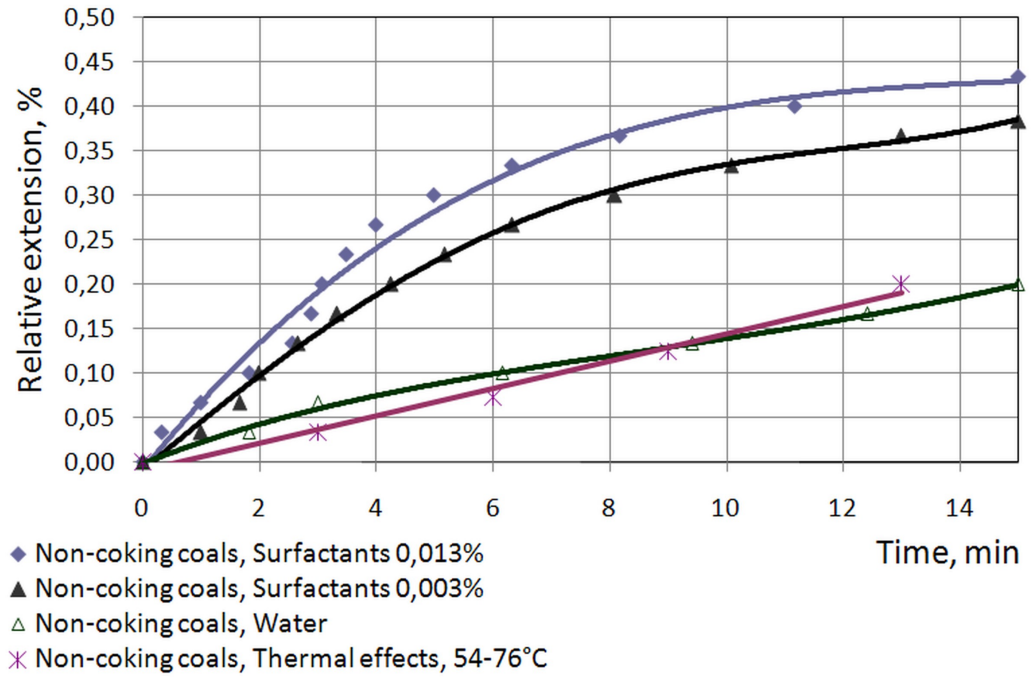

Fig. 4. Expansion of coal with wetting and thermal effects.

The use of surfactants, the "Elfor" type wetters, used in mining industry for wetting, leads to an increase in the self-movement velocity of the fluid under the action of capillary forces by a factor of 2-3. This simultaneously affects the rate of deformation of pore space, due to wedging, swelling as a result of interfacial processes at the interface.

\section{Conclusions}

The filtration process in coal in our ideas is a multistage process, accompanied by a change in the properties and state of coal, dispersed components - liquid and gas. Filtration of liquid and gas inside the pore space is accompanied not only by the mass transfer of these phases, but also by simultaneously changing the properties of the surface of the solid phase and the structure of the capillary-porous space.

If at the initial moment in the hydrophobic state of the surface, filtration is impossible, then after the hydophilization of the coal surface, the filtration pattern reverses. This determines the optimal maximum for the filtration time of the liquid, at which the filtration rate has a maximum value.

Being in a stress-strain state, in which the porosity is stress-dependent, interfacial interaction, swelling, wedging leads to deformation of pore space, as a result, the intensity of the decrease in filtration rate increases several times, that consistently leads to a decrease in the efficiency of hydro treatment. 


\section{References}

1. O. I. Chernov, V.Y. Al'perovich, Principles of integrated methods to fight coal dust, gassing, sudden coal and gas, rock bursts and endogenous fires in coal mines (Book Publishers, Kemerovo, 1961)

2. O. I. Chernov, E.S. Rozantsev, Preparation of mine fields with gazovybrosoopasnymi layers (Nedra, Moscow, 1975)

3. V. G. Smirnov, V. V. Dyrdin, A. Yu. Manakov, Z. R. Ismagilov, The problem of formation of pulverized coal at mine outburst causd by methane hydrates (KuzSTU, Kemerovo, 2016)

4. The federal rules and regulations in the field of industrial safety "Instructions Anti-dust in coal mines" (RIS, Moscow, 2014)

5. By Jay F. Colinet, J. P. Rider, J. M. Listak, J. A. Organiscak, A. L. Wolfe, Best Practices for Dust Control in Coal Mining (DHHS, Pittsburgh, 2010)

6. S. N. Roychowdhury, Coal dust explosions and their prevention (Missouri University of Science and Technology, Rolla, 1960)

7. S. A. Shepeleva, V. V. Dyrdin, J. Min. Sci., 47, 5 (2011)

8. I. S. Elkin, Coal in the 21st Century, Mining, Intelligent Equipment and Environment Protection (AP, Qingdao, 2018)

9. I. S. Elkin, A. Meshkov, E3S Web Conf., 21, 01023 (2018)

10. H. Darcy, Les fontaines publiques de la ville de Dijon (Dalmont, Paris, 1856)

11. S Whitaker, J. Transport in Porous, 1, 3 (1986) 